\title{
CONCERNING THE ZEROS OF THE SOLUTIONS OF CERTAIN
}

\section{DIFFERENTIAL EQUATIONS*}

\author{
BY \\ WILLIAM BENJAMIN FITE
}

In volume 42 of the $\mathrm{Mathematische} \mathrm{Annalen} \mathrm{Kneser} \mathrm{has}$ shown that the solutions of equations of the form $y^{(n)}+q y=0$ oscillate an infinite number of times, provided that $x^{m} q>k>0$ for sufficiently great values of $x$, where $n \geqq 2 m>0$ and $n$ is even. In case $n$ is odd the solutions either oscillate an infinite number of times or approach zero together with their first $n$ derivatives. In Section I of this paper the oscillation of solutions of certain linear equations of the second order is discussed and some results are obtained which are not included in Kneser's. Linear equations of a certain trinomial form are discussed in Section II, and in Section III Kneser's conclusions are shown to hold when $m$ is restricted merely to being less than $n$. A condition of a slightly different nature; namely, that the integral $\int_{x_{1}}^{x} q d x$ diverge, is also shown to be sufficient to insure the oscillation of the solutions an infinite number of times, except possibly when $n$ is odd. It is worth while observing that not only must the solutions vanish an infinite number of times, as Kneser says, but they must change sign an infinite number of times. Some of the conclusions of this section are also applicable to certain non-linear equations. Finally, in Section IV, the solutions of systems of linear equations are considered. It is shown as a particular case of a more general result that under specified conditions not all the functions which form a solution can vanish within an interval whose length is less than an assigned value.

Consider the equation

$$
y^{\prime \prime}+p y^{\prime}+q y=0,
$$

where, for $x \geqq x_{1},|p| \leqq M$, and $q \geqq h>0, M$ and $h$ being constants such that $4 h-M^{2}>0$.

If (1) had a solution $y$ that vanished only a limited number of times for $x>x_{1}$, and if $y^{\prime}$ were not negative or zero for all values of $x$ greater than a

\footnotetext{
* Presented to the Society October 27 and December 27, 1917.
} 
certain $x_{2}$, we could take a number $c\left(>x_{2}\right)$ such that $y^{\prime}(c)>0$ and consider the solution $y_{2}$ of the equation

$$
y^{\prime \prime}-M y^{\prime}+h y=0,
$$

whose derivative vanishes for $x=c$. We can choose $x_{2}$ sufficiently great to insure that $y_{2}=0$ for a value $a(<c)$ of $x$ greater than the greatest root of $y$. If $y_{1}$ is the solution of (1) such that $y_{1}(a)=0$, then $y_{1}^{\prime}(x)=0$ for $a<x \leqq c .^{*}$ There is therefore a solution $y_{3}$ of (1) such that $y_{3}^{\prime}(c)=0$ and $y_{3}(x)=0$ for $a \leqq x<c$. We can assume that $y(c)=y_{3}(c)$. For values of $x$ a little less than $c, y(x)<y_{3}(x)$. Therefore $y(x)=y_{3}(x)$ for some value of $x$ between $a$ and $c$. But this would give a solution, $y-y_{3}$, of (1) which vanished twice in $(a, c)$. Since this is impossible, we can assume $y^{\prime}(x) \leqq 0$ for all sufficiently great values of $x$.

Consider now a solution $y_{4}$ of the equation

$$
y^{\prime \prime}+M y^{\prime}+h y=0
$$

such that $y_{4}^{\prime}(c)=0$, where $c$ is such that $y^{\prime}(c)<0$. Now $y_{4}(b)=0$ for some $b$ greater than $c$ and the solution of (1) that vanishes for $x=b$ has a derivative that vanishes in $(c, b) . \dagger$ Hence if $y_{5}$ is the solution of (1) such that $y_{5}^{\prime}(c)=0$, then $y_{5}(x)=0$ for some $x$ in $(c, b)$. We could then assume that $y(c)=y_{5}(c)$. But this would require that $y(x)=y_{5}(x)$ for some $x$ in $(c, b)$ exclusive of $c$. This is, however, impossible and we have therefore completed the proof of

THEOREM I. If in equation (1) $p$ and $q$ are continuous functions of $x$ such that $|p| \leqq M$ and $q \geqq h>0$ for every $x \geqq x_{1}$, where $M$ and $h$ are constants such that $4 h-M^{2}>0$, then every solution changes sign an infinite number of times. $\neq$

In his memoir on Linear Differential Equations of the Second Order§ Sturm considered the effect produced upon the zeros of the solutions of (1) by certain changes in the coefficients. These changes however were not of the kind considered in the following theorem since they did not involve a simultaneous increase in $p$ and $q . \|$

Theorem II. If in the equations

(1)

and

(4)

$$
y^{\prime \prime}+p y^{\prime}+q y=0
$$

$$
y^{\prime \prime}+p_{1} y^{\prime}+q_{1} y=0
$$

*Annals of Mathematics, vol. 18 (1917), p. 216.

† Annals of Mathematics, loc. cit., Theorem II, p. 216.

†Cf. Kneser, Journal für die reine und angewandte Mathematik, vol. 117 (1897), p. 80.

§Journalde Mathématiques, vol. 1 (1836), p. 106.

$\|$ Cf. Annals of Mathematics, loc. cit., p. 216. 
$p, q, p_{1}$, and $q_{1}$ are continuous functions of $x$ such that $p_{1} \leqq p \leqq 0, q_{1} \leqq q$, and $q>0$, for all values of $x$ greater than $x_{1}$, and if every solution of (4) vanishes an infinite number of times for these values of $x$, then every solution of (1) vanishes an infinite number of times.

Let $y_{2}$ be the solution of (4) that vanishes for $x=a>x_{1}$. Then since by hypothesis $y_{2}$ vanishes an infinite number of times for $x>a$, there is a number $b(>a)$ such that $y_{2}^{\prime}(b)=0$. If now $y$ is the solution of (1) that vanishes for $x=a$, then $y^{\prime}(c)=0$ and $y(c)>0$, where $a<c \leqq b$. $^{*}$ Now $y^{\prime \prime}(c)=-q(c) y(c)<0$. If $y^{\prime \prime}(x)$ remained negative for all values of $x$ greater than $c, y$ would vanish for some such $x$. If $y^{\prime \prime}(x)$ did not remain negative, it would become zero. Let $d$ be the first value of $x>c$ for which $y^{\prime \prime}(x)=0$. Then $p(d) y^{\prime}(d)+q(d) y(d)=0$. But $p(d) \leqq 0$ by hypothesis and $y^{\prime}(d)<0$ since $y^{\prime}(c)=0$ and $y^{\prime \prime}(x) \leqq 0$ in the interval $(c, d)$. Hence this last equation is an impossible one, unless $y$ vanishes in the interval $(c, d)$. Therefore $y(x)$ has one root, and hence an infinite number of roots, greater than $a$.

The assumption in the theorem that every solution of (4) vanishes an infinite number of times for $x>x_{1}$ is more restrictive than it need be. It would be sufficient to assume that for these values of $x$ every root of every solution is followed by a root of the derivative of this solution.

It is natural to inquire whether the restriction that $p \leqq 0$ is a necessary one. As a partial answer to the question we observe that some restriction on $p$ is certainly necessary, since no solution of the equation

$$
y^{\prime \prime}+3 y^{\prime}+2 y=0
$$

vanishes more than once, although every solution of the equation

$$
y^{\prime \prime}+y^{\prime}+y=0
$$

has an infinite number of roots greater than any assigned number.

If we change the dependent variable from $y$ to $\bar{y}$ by means of the relation $y=x^{\lambda} \bar{y}$, where $\lambda$ is a constant, we get

If now

$$
\bar{y}^{\prime \prime}+\left(p+\frac{2 \lambda}{x}\right) \bar{y}^{\prime}+\left(\frac{\lambda^{2}-\lambda}{x^{2}}+\frac{\lambda p}{x}+q\right) y=0 .
$$

$$
\frac{\lambda^{2}-\lambda}{x^{2}}+\frac{\lambda p}{x}+q \leqq 0
$$

for $x \geqq x_{1}>0$, no $\bar{y}$, and therefore no $y$, can vanish more than once for these values of $x$. Various sufficient conditions for the non-vanishing of $y$ for more than a finite number of values of $x$ greater than $x_{1}$ can be obtained from (5) by assigning particular values to $\lambda$.

* Annals of Mathematics, loc. cit. 
Consider the equation

$$
y^{(n)}+p y^{(n-1)}+q y=0,
$$

where $p$ and $q$ are continuous functions of $x$ in the interval considered and $q$ does not vanish. In counting the number of times a function vanishes each zero will be counted a number of times equal to its multiplicity.

If $y^{(n-1)}(x)=0$ and $y(x) \neq 0$, the sign of $y^{(n)}(x)$ is opposite that of $q(x) y(x)$. Hence $y^{(n-1)}$ cannot vanish twice within any interval within which $y$ does not vanish, and therefore $y^{\prime}$ cannot vanish $n$ times within any such interval.

If $q>0$ for $x \geqq x_{1}$, a zero of $y$ in this interval followed by a sequence of $n-1$ zeros of $y^{\prime}$ without a zero of $y$ must be followed by a zero of $y$ in case $n$ is greater than 2. For $y^{(n-1)}$ has just one zero in this interval, say $x=a$, and must therefore be negative for values of $x$ greater than $a$, since $y^{(n)}(a)$ is negative. Moreover $y^{(n-2)}$ must have a zero which is equal to, or greater than, $a$, and for all values of $x$ greater than this zero $y^{(n-2)}$ and $y^{(n-1)}$ would both be negative if $y$ did not vanish. But this is impossible. The exception for $n=2$ is an exception in fact, as may be seen from a consideration of the equation $y^{\prime \prime}+3 y^{\prime}+2 y=0$. The solution $y=e^{-x}-e^{-2 x}$ has the single zero $x=0$, and $y^{\prime}=-e^{-x}+2 e^{-2 x}$ vanishes for $x=\log 2$.

If $q<0$ in an interval, a sequence of $n-1$ zeros of $y^{\prime}$ without a zero of $y$ cannot be followed by a zero of $y$. For if this were possible $y^{(n-1)}$ would change from zero to a negative value while $y^{(n)}$ is positive. But there must be an odd number of zeros of $y^{\prime}$ between successive zeros of $y$. Hence if $n$ is even and $q<0$, there cannot be more than $n-3$ zeros of $y^{\prime}$ between successive zeros of $y .^{*}$

If $n$ is odd and $q$ does not vanish, there cannot be more than $n-2$ zeros of $y^{\prime}$ between successive zeros of $y$. If $q>0$ for $x \geqq x_{1}$ and a zero of $y$ in this interval is followed by a sequence of $n-2$ zeros of $y^{\prime}$ without a vanishing of $y, y^{(n-2)}$ must change from a positive to a negative value, and therefore $y^{(n-1)}$ must be negative when $y^{(n-2)}$ is negative. But $y^{(n-1)}$ cannot change from a negative value to zero while $y$ is positive since when $y^{(n-1)}$ is zero $y^{(n)}$ is negative. If then $y$ remained positive, $y^{(n-1)}$ and $y^{(n-2)}$ would remain negative. But this is impossible. Hence if $n$ is odd and $q>0$ for $x \geqq x_{1}, a$ zero of $y$ in this interval followed by a sequence of $n-2$ zeros of $y^{\prime}$ without a zero of $y$ must be followed by a zero of $y$.

Suppose now that, for $x \geqq x_{1}, p$ and $q$ are continous functions of $x$ such that $p \geqq 0, q \geqq h>0$, and $p$ is bounded. If for any value of $x$ under considera-

* For the case $n=2$ see Bôcher, these Trans a ction s, vol. 3 (1902), p. 199. Cf. also Kneser, Journal für reine und angewandte Mathematik, vol. 116 (1896), p. 181; M a the mat is che An a le n, vol. 42 (1893), p. 413. 
tion $y$ and $y^{(n-1)}$ are both positive, $y^{(n)}$ must be negative. Then $y^{(n-1)}$ must decrease as $x$ increases. If $y$ does not vanish as $x$ increases indefinitely and $y^{(n-1)}$ remains positive, it is possible to select values of $x$ as great as we please for which the absolute value of $y^{(n)}$ is as small as we please. Therefore $y$ will be as small as we please for indefinitely great values of $x$. But $y^{\prime}$ can vanish only a limited number of times for $x \geqq x_{1}$. Hence $y$ must approach zero. If on the other hand, $y^{(n-1)}$ is zero for some value of $x$ for which $y$ is positive, it will be negative for slightly greater values of $x$. But it cannot increase from a negative value to zero if $y$ remains positive since when it is zero $y^{(n)}$ is negative. And if it remains negative, $y^{(n-2)}$ must steadily decrease. If as $x$ increases indefinitely $y$ does not vanish and $y^{(n-2)}$ does not become negative, it is possible to select values of $x$ as great as we please for which the absolute value of $y^{(n-1)}$ is as small as we please. Then since $p$ is bounded it is possible to select values of $x$ as great as we please for which $p y^{(n-1)}$ is as small as we please in absolute value and $y^{(n)}$ is positive. This requires that $y$ come as near to zero as we please, and therefore that $y$ approach zero as a limit. If $y^{(n-2)}$ should become negative it would remain negative and $y$ would ultimately vanish. This completes the proof of

Theorem III. If in equation (6), for $x \geqq x_{1}, p$ and $q$ are continuous functions of $x$ such that $p \geqq 0, q \geqq h>0$, and $p$ is bounded, every solution either vanishes an infinite number of times or approaches zero as $x$ increases indefinitely.

\section{III}

We consider now equations of the form

$$
y^{(n)}+q y=0,
$$

where $q$ is a continuous function of $x$ for $x \geqq x_{1}$. For any number $a \geqq x_{1}$ and any solution $y$ of (7) we have

$$
\begin{aligned}
y(x)=y(a)+y^{\prime}(a)( & -a)+\cdots \\
& +y^{(n-1)}(a) \frac{(x-a)^{n-1}}{(n-1) !}+y^{(n)}\left(x_{0}\right) \frac{(x-a)^{n}}{n !},
\end{aligned}
$$

where $x \geqq x_{1}, x_{0}=a+\theta(x-a)$, and $0<\theta<1$. If now the equation

$$
y(a)+y^{\prime}(a)(x-a)+\cdots+y^{(n-1)}(a) \frac{(x-a)^{n-1}}{(n-1) !}=0
$$

is satisfied by a positive value of $x-a$, say $b-a$, then $y(x)=0$ for some $x$ between $a$ and $b$, provided that $q>0$. For if this were not the case we could assume $y>0$ for $a<x \leqq b$, and if we put $x=b$ in (8) the left member would be positive while the right member would be negative by virtue of (7) 
and (9). If, on the other hand, $q<0$ and $y(a) \geqq 0$, the first root $b$ of $y(x)=0$ that is greater than $a$ must be such that $b-a$ exceeds the least positive value of $x-a$ that satisfies (9). In particular, if no positive value of $x-a$ satisfies (9), $y$ cannot vanish for any value of $x$ greater than $a$.

We get immediately from (7)

$$
y^{(n-1)}(x)=-\int_{x_{1}}^{x} q y d x+y^{(n-1)}\left(x_{1}\right)
$$

If now $y$ is greater than, or equal to, zero for $x \geqq x_{1}$ and $q \geqq 0$, the integral $\int_{x_{1}}^{x} q y d x$ cannot diverge as $x$ increases indefinitely. For if it did, $y^{(n-1)}$ would become negative, and, since $y^{(n)}$ is by hypothesis negative or zero, $y$ would become negative, contrary to the hypothesis.

Theorem IV. If $q \geqq 0$ when $x \geqq x_{1}$ and $y$ is a solution of (7) such that the integral $\int_{x_{1}}^{x} q y d x$ diverges as $x$ increases indefinitely, $y$ must change sign an infinite number of times.

In case $q \leqq 0$ any solution that does not change sign an infinite number of times must become infinite if the integral diverges, since $y^{(n-1)}$ becomes and remains greater than any given positive number.

A direct application of Theorem IV shows that if the integral $\int_{x_{1}}^{x} q d x$ diverges there can be no solution $y$ which exceeds a given positive number $h$ for $x \geqq x_{1}$. Moreover if $y$ does not change sign $y^{\prime}$ cannot vanish more than $n-1$ times. Hence any solution which does not change sign an infinite number of times must approach zero as $x$ increases indefinitely.

Now we have already made use of the fact that if two consecutive derivatives of $y$ are negative or zero for all values of $x$ greater than a given number $a, y$ must ultimately be negative; and if two consecutive derivatives are positive or zero for these values of $x, y$ must become positively infinite with $x$. Moreover if we assume that $y$ is not negative for $x$ greater than $a, y^{(n)}$ must be negative or zero for these values of $x$. Hence if $y^{(n-1)}$ were negative or zero for any such value of $x$ it would be negative for all greater values and $y$ would change sign. We must assume then that $y^{(n-1)}$ is positive when $x$ is greater than $a$. If $y^{(n-2)}$ were positive or zero for such a value of $x$, it would be positive for all greater values and $y$ would become infinite with $x$. We must assume then that $y^{(n-2)}$ is negative when $x$ is greater than $a$. By continuing this argument we conclude that when $n$ is even $y^{\prime \prime}$ must be negative for these values of $x$. On the other hand, the supposition that $y$ approaches zero requires that $y^{\prime}$ become negative and hence the supposition that $y$ does not change sign for $x$ greater than $a$ is untenable.

When $n$ is odd this argument leads to the conclusion that if $y$ does not change sign an infinite number of times, $y^{\prime}$ must become and remain negative. But this is not inconsistent with the fact that $y$ approaches zero as $x$ increases 
indefinitely,* as may be seen from the function $y=e^{-x}$, which is a solution of (7) when $n$ is any positive odd integer and $q \equiv 1$. If however $y$ vanishes at all for $x \geqq x_{1}$ it must change sign an infinite number of times, since otherwise it would approach zero and $y^{\prime}$ would change sign from positive to negative. This would require $y^{\prime \prime}$ to be negative for some value of $x$ greater than $x_{1}$. It would then be negative for all greater values of $x$ and $y$ would change sign. This completes the proof of

Theorem V. If $q>0$ and the integral $\int_{x_{1}}^{x} q d x$ diverges as $x$ increases indefinitely every solution of (7) must change sign an infinite number of times for $x \geqq x_{1}$ in case $n$ is even, and in case $n$ is odd such a solution must either change sign an infinite number of times or not vanish at all for $x \geqq x_{1} \cdot \dagger$

Suppose now that we have

$$
x^{m_{1}} q>h>0
$$

for $x \geqq x_{1}$. This inequality will be strengthened if we replace $m_{1}$ by $m$, where $m>m_{1}$. We can so select $m$ that $1 /(n-m)$ is an integer greater than 1 , whatever value $m_{1}$ may have less than $n$. If $y$ did not change sign an infinite number of times or ${ }^{\circ}$ did not approach zero as $x$ increases indefinitely, there would be a positive number $h_{1}$ such that $y(x)>h_{1}$ for sufficiently great values of $x$. We proceed to prove that this is impossible and that therefore $y$ must either change sign an infinite number of times or approach zero as $x$ increases indefinitely. Moreover it follows from the proof of Theorem V that the latter can occur only under certain circumstances when $n$ is odd.

We take $x_{1}$ sufficiently great so that $y(x)>h_{1}$ for $x \geqq x_{1}$ and consider the consequences of the inequality

$$
y^{(n)}(x)<-\frac{k}{x^{m}}
$$

where $k=h h_{1}>0$. From (10) we get

If

$$
y^{(n-1)}(x) \leqq \frac{k}{m-1}\left(\frac{1}{x^{m-1}}-\frac{1}{x_{1}^{m-1}}\right)+y^{(n-1)}\left(x_{1}\right)
$$

$$
y^{(n-1)}\left(x_{1}\right)-\frac{k}{(m-1) x_{1}^{m-1}}<0 \text {, }
$$

$y^{(n-1)}(x)$ would be negative for sufficiently great values of $x$ and $y$ would become negative. We assume therefore that if $n>1$

$$
y^{(n-1)}\left(x_{1}\right)-\frac{k}{(m-1) x_{1}^{m-1}} \geqq 0 .
$$

${ }^{*}$ In this case $y^{\prime}, y^{\prime \prime}, \cdots, y^{(n-1)}$ all approach zero. Cf. Kneser, loc. cit., vol. 42, p. 435.

$\dagger$ Cf. the result given by Kneser, loc. cit., p. 420.

Trans. Am. Math. Soc. 23 
Moreover if the expression in the left member of this inequality should become negative as $x_{1}$ increases, we could apply the preceding argument for a properly chosen $x_{1}$. There remains then to be considered the case in which

$$
y^{(n-1)}(x)-\frac{k}{(m-1) x^{m-1}} \geqq 0
$$

for $x \geqq x_{1}$. From this inequality we get

$$
y^{(n-2)}(x) \geqq \frac{k}{(m-1)(m-2)}\left(\frac{1}{x_{1}^{m-2}}-\frac{1}{x^{m-2}}\right)+y^{(n-2)}\left(x_{1}\right) .
$$

If $n>2$ and $y^{(n-2)}(x)$ is to remain negative as $x$ increases we must have

Hence

$$
y^{(n-2)}(x)+\frac{k}{(m-1)(m-2) x^{m-2}} \leqq 0 .
$$

$$
y^{(n-3)}(x) \leqq \frac{k}{(m-1)(m-2)(m-3)}\left(\frac{1}{x^{m-3}}-\frac{1}{x_{1}^{m-3}}\right)+y^{(n-3)}\left(x_{1}\right) .
$$

Now if $y$ does not change sign as $x$ increases, no derivative of index $n-2 i-1$ can become negative if all the derivatives with indices of the form $n-2 j$, where $j \leqq i$, remain negative. There are therefore but two conceivable results of a continuation of this argument:

(a) The derivatives of $y$ from the nth to the first inclusive are of alternate signs for $x \geqq x_{1}$. In case $n$ is odd this supposition leads to the inequality

$$
y(x) \leqq \frac{-k}{(m-1)(m-2) \cdots(m-n)}\left(x_{1}^{n-m}-x^{n-m}\right)+y\left(x_{1}\right) .
$$

From this we conclude that $y$ must become negative.

When $n$ is even the situation is not so simple. In this case we have

Hence

$$
y^{\prime}(x) \geqq \frac{k}{(m-1)(m-2) \cdots(m-n+1) x^{m-n+1}} .
$$

$$
y(x) \geqq \frac{-k}{(m-1) \cdots(m-n+1)(m-n)}\left(x^{n-m}-x_{1}^{n-m}\right)+y\left(x_{1}\right) .
$$

There is therefore a positive number $l_{1}$ such that $y(x)>l_{1} x^{n-m}$ for suffciently great values of $x$, and we can replace (10) by

$$
y^{(n)}(x)<\frac{-k_{1}}{x^{2 m-n}} \quad\left(k_{1}>0\right) .
$$

This in turn gives us $y(x)>l_{2} x^{2(n-m)}\left(l_{2}>0\right)$, and we can accordingly replace (10) by

$$
y^{(n)}(x)<-\frac{k_{2}}{x^{3 m-2 n}} \quad\left(k_{2}>0\right) .
$$


A continuation of this process leads finally to the relation

$$
y^{(n)}(x)<\frac{-k_{r}}{x^{n-1}} \quad\left(k_{r}>0\right),
$$

which in turn gives us

$$
y^{\prime}(x) \leqq \frac{k_{r}}{(n-2) !} \log \frac{x_{1}}{x}+y^{\prime}\left(x_{1}\right) .
$$

But this is contrary to (11).

(b) The derivative of index $n-2 i$ is positive for sufficiently great values of $x$, while all the derivatives with indices of the form $n-2 j(0 \leqq j<i)$ are negative for $x \geqq x_{1}$ and all the derivatives with indices of the form $n-2 j+1(1 \leqq j \leqq i)$ are positive for these values of $x$.

Since $y^{(n-2 i)}(x)$ is positive and increasing, we should have $y(x)>l_{1} x^{n-2 i}$ $\left(l_{1}>0\right)$ for sufficiently great values of $x$. We could therefore replace (10) by

$$
y^{(n)}(x)<\frac{-k_{1}}{x^{m-n+2 i}} \quad\left(k_{1}>0\right) .
$$

But a series of steps similar to those described under $(a)$ shows that this inequality requires that $y^{(n-2 i+1)}(x)$ be negative for sufficiently great values of $x$. This however contradicts our assumption.

This completes the proof of

TheOREM VI. If in the equation

$$
y^{(n)}+q y=0
$$

$q$ is a positive infinitesimal of order less than $n$ with respect to $1 / x\left(x \geqq x_{1}\right), y$ must change sign an infinite number of times as $x$ increases from $x_{1}$, in case $n$ is even. If $n$ is odd $y$ must change sign an infinite number of times as $x$ increases from $x_{1}$ or not vanish at all for $x \geqq x_{1}$.

The restriction that $m<n$ is essential since such an equation as

$$
y^{\text {iv }}+\frac{15 y}{16 x^{4}}=0,
$$

for example, has $y=x^{5 / 2}$ for a solution.

Some of the foregoing results can be extended to certain non-linear equations of the form

$$
y^{(n)}+f(x, y)=0 .
$$

Suppose that $f(x, y)$ has the following properties:

1. It is continuous for $x$ and $y$ finite and $x \geqq x_{1}$.

2. When $x \geqq x_{1}$, it is positjve or negative according as $y$ is positive or negative. 
3. For any $x$ ( $\left.\geqq x_{1}\right)$ and any two finite values of $y$, as $y_{1}$ and $y_{2}$,

$$
\left|f\left(x, y_{1}\right)-f\left(x, y_{2}\right)\right|<A\left|y_{1}-y_{2}\right|
$$

where $A$ is a constant.

No solution has a singular point for any finite value of $x \geqq x_{1}$. If a solution $y$ does not change sign an infinite number of times as $x$ increases from $x_{1}$ it must ultimately either increase monotonically or decrease monotonically, since $y^{\prime}$ cannot vanish more than $n-1$ distinct times within an interval within which $y$ does not vanish. But $y$ can approach zero monotonically only in case it does not vanish at all for $x \geqq x_{1}$ and $n$ is odd. A repetition of the analysis used in the proof of Theorem IV shows that, if $y$ is such that the integral $\int_{x_{1}}^{x} f(x, y) d x$ diverges as $x$ increases without limit, it must change sign an infinite number of times.

\section{IV}

In the Annals of $\mathrm{M}$ athem at ics for June, 1917, I have established the existence of a minimum length for an interval within which any solution of a linear homogeneous differential equation of order $n$ and its first . $n-1$ derivatives can all vanish. This raises the question as to the existence of such minimum intervals for the solutions of more general classes of equations. I show here that these minimum intervals do exist for systems of equations of the first order subject to certain restrictions. In fact a somewhat more general result is established from which the existence of the intervals in question can be inferred as a special case.*

Consider the system of equations

$$
\begin{aligned}
& \frac{d y_{1}}{d x}=f_{1}\left(x, y_{1}, y_{2}, \cdots, y_{n}\right), \\
& \frac{d y_{2}}{d x}=f_{2}\left(x, y_{1}, y_{2}, \cdots, y_{n}\right) \text {, } \\
& \frac{d y_{n}}{d x}=f_{n}\left(x ; y_{1}, y_{2}, \cdots, y_{n}\right) \text {, }
\end{aligned}
$$

where the $f^{\prime}$ 's are continuous functions of their arguments when $x$ is in the interval $(a, b)$ and $y_{1}, y_{2}, \cdots, y_{n}$ are finite. Moreover the inequalities

$$
\begin{gathered}
\left|f_{i}\left(x, y_{1}, y_{2}, \cdots, y_{n}\right)-f_{i}\left(x, z_{1}, z_{2}, \cdots, z_{n}\right)\right|<\sum_{k=1}^{n} L_{k}\left|y_{k}-z_{k}\right|, \\
(i=1,2, \ldots, n)
\end{gathered}
$$

\footnotetext{
* This more general result was not contained in the paper presented to the Society. It was suggested to me later by Dr. J. F. Ritt.
} 
where $L_{1}, L_{2}, \cdots, L_{n}$ are certain positive constants, are supposed to be satisfied for any finite values of $y_{k}$ and $z_{k}$.

Let $y_{1}, y_{2}, \cdots, y_{n}$ and $z_{1}, z_{2}, \cdots, z_{n}$ be two solutions of these equations such that each of the differences $y_{i}-z_{\downarrow}$ vanishes somewhere in $(a, b)$. Picard's method of successive approximations, which is applicable to this case, shows that the functions $y_{i}$ and $z_{i}$ remain finite within $(a, b)$. The difference $y_{i}-z_{i}$ has therefore a maximum absolute value which we shall represent by $M_{i}$. Now

$$
\begin{aligned}
\left|\frac{d\left(y_{i}-z_{i}\right)}{d x}\right|=\mid f_{\iota}\left(x, y_{1}, y_{2}, \cdots, y_{n}\right)-f_{i}\left(x, z_{1}, z_{2}, \cdots\right. & \left., z_{n}\right) \mid \\
& <\sum_{k=1}^{n} L_{k}\left|y_{k}-z_{k}\right| .
\end{aligned}
$$

Moreover $y_{i}-z_{i}=0$ somewhere within $(a, b)$. Hence

where $b-a=\rho$.

$$
M_{i}<\sum_{k=1}^{n} L_{k} M_{k} \cdot \rho,
$$

If in this inequality we give to $i$ the values $1,2, \cdots, n$ in turn and multiply the resulting inequalities by $L_{1}, L_{2}, \cdots, L_{n}$ respectively, and add, we get, after dividing by $\sum L_{k} M_{k}$,

$$
1<\sum L_{k} \rho,
$$

or

$$
\rho>\frac{1}{\sum \overline{L_{k}}} .
$$

We have therefore

THEOREM VII. If the corresponding functions of two solutions of equations $A$ are equal somewhere within the interval $(a, b)$, the length of this interval must exceed $1 / \sum L_{k}$.

If we assume further that the functions $f_{i}$ are such that

$$
f_{i}(x, 0,0, \cdots, 0)=0
$$

within $(a, b)$, then $0,0, \cdots, 0$ is a solution of $A$ and we can apply Theorem VII to this and the solution $y_{1}, y_{2}, \cdots, y_{n}$. This leads to the conclusion that if $\rho \leqq 1 / \sum L_{k}$ no non-identically variishing solution $y_{1}, y_{2}, \cdots, y_{n}$ of equations $A$ in which $f_{i}(x, 0,0, \cdots, 0)=0$ within an interval $(a, b)$ of length $\rho$ can be such that all the $y$ 's vanish within $(a, b)$.

This conclusion can not be drawn from the conditions of Theorem VII, as may be seen from a consideration of the system

$$
\begin{aligned}
& \frac{d y_{1}}{d x}=\frac{e^{x}}{3}-y_{1}-\frac{2}{3} y_{2}, \\
& \frac{d y_{2}}{d x}=-x+\frac{4}{3} y_{1}+y_{2} .
\end{aligned}
$$


This system satisfies all the conditions of the theorem for any finite interval, but not the condition that $f_{i}(x, 0,0, \cdots, 0)=0$. On the other hand, it has the solution

$$
\begin{aligned}
& y_{1}=\left(6 e^{1 / 3}+\frac{1}{2} e^{-2 / 3}\right) e^{x / 3}-\left(12 e^{-1 / 3}+\frac{1}{2} e^{-4 / 3}\right) e^{-x / 3}-6 x, \\
& y_{2}=-\left(12 e^{1 / 3}+e^{-2 / 3}\right) e^{x / 3}+\left(12 e^{-1 / 3}+\frac{1}{2} e^{-4 / 3}\right) e^{-x / 3}+\frac{1}{2} e^{x}+9 x+9
\end{aligned}
$$

and $y_{1}$ and $y_{2}$ both vanish when $x=-1$.

Analogous results hold in case the $f$ 's are analytic functions of the complex arguments $x, y_{1}, y_{2}, \cdots, y_{n}$ when $x$ remains within the circle of center $x_{0}$ and radius $r$ (or upon its circumference) and $y_{1}, y_{2}, \cdots, y_{n}$ are finite, subject to the condition that for any fixed $k$ from 1 to $n$ inclusive and every $i$ from 1 to $n$ inclusive

$$
\left|\frac{\partial f_{i}}{\partial y_{k}}\right|<L_{k}
$$

for these values of the argument.

Columbia University 